\title{
Caracterización del nivel de razonamiento científico en futuros profesores: desafíos para la formación inicial docente
}

\author{
Gonzalo R. Guerrero( ${ }^{(1)}$, Silvia Tecpan ${ }^{(2)}$, Sandra P. Rojas-Rojas ${ }^{(3)}$ y Carol L. Joglar (4) \\ (1) Institute of Education, University College London, Londres, Reino Unido. (correo-e: g.hernandez@ucl.ac.uk) \\ (2) Facultad de Ciencias, Departamento de Física, Universidad de Santiago de Chile, Santiago, Chile. \\ (correo-e: silvia.tecpan@usach.cl) \\ (3) Facultad de Química y Biología, Departamento de Ciencias del Ambiente, Universidad de Santiago de Chile, \\ Santiago, Chile. (correo-e: sandra.rojas.r@usach.cl) \\ (4) Facultad de Química y Biología, Departamento de Biología, Universidad de Santiago de Chile, Santiago, Chile. \\ (correo-e: carol.joglar@usach.cl)
}

Recibido Dic. 24, 2019; Aceptado Feb. 28, 2020; Versión final Abr. 9, 2020, Publicado Oct. 2020

\begin{abstract}
Resumen
El objetivo de este estudio fue determinar la relación entre el nivel de razonamiento científico (NRC) y variables académicas de pre-ingreso de 158 estudiantes de primer año en cinco carreras de pedagogía en una universidad pública chilena. Este estudio forma parte de los instrumentos que contribuyen a la evaluación inicial nacional diagnóstica considerando los nuevos requerimientos de la ley de carrera docente. La metodología se enmarcó en un estudio transeccional correlacional-causal. Para diagnosticar el NRC, se aplicó el Test de Lawson adaptado al contexto chileno y se diseñaron modelos de regresión multi-variable. Los resultados muestran la incidencia en el NRC de la variable sexo y variables académicas de pre-ingreso como puntajes en Prueba de Selección Universitaria (PSU) en Ciencias y Lenguaje; como también vías de acceso alternativas e inclusivas a las carreras de pedagogía. Se concluye que los niveles de razonamiento científico de los estudiantes que ingresan a carreras de pedagogía de primer año se ubican principalmente en niveles transicionales.
\end{abstract}

Palabras clave: razonamiento científico, formación inicial docente, diagnóstico, educación superior, formación docente

\section{Characterisation of scientific reasoning levels among pedagogy freshman students: challenges for initial teacher education}

\begin{abstract}
The aim of this study was to determine the relationship of the level of scientific reasoning (LSR) and preadmission academic variables of 158 freshman students from five teaching programs in a Chilean public university. This study is part of a group of instruments that contribute to the national diagnosis for new requirements of law teaching career in Chile. The methodology was part of an ex post-facto transeccional causal-correlational study. The Lawson Test, adapted to the Chilean context, and multi-variable regression models were applied and designed to diagnose the LSR. The results showed the incidence in the LSR of the sex variable and pre-admission academic variables such scores in science and language in the Chilean university selection test (known as PSU). The results also showed alternative and inclusive access routes to teaching careers. It is concluded that level of scientific reasoning among first year students enrolled in teaching programs were primarily at transitional levels.
\end{abstract}

Keywords: scientific reasoning, initial teacher training, diagnosis, higher education, teacher education 


\section{INTRODUCCIÓN}

Actualmente las instituciones de educación superior (IES), en particular las universidades, se enfrentan al desafío de generar y promover la formación inclusiva de estudiantes altamente heterogéneos (UNESCO, 2017). Asimismo, y en específico, las carreras de pedagogía están migrando a nuevos sistemas de acceso que buscan favorecer la equidad e inclusión de la diversidad del estudiantado que ingresa a las aulas universitarias (Infante, 2009; Zárate-Rueda et al., 2017). Este escenario, trae consigo la responsabilidad institucional de generar acciones que contribuyan a diagnosticar y acompañar la trayectoria académica de los estudiantes desde el primer momento en que ingresan a la universidad hasta su egreso. En el caso de Chile, las IES que forman profesores se rigen por la Ley № 20.129 del año 2018, la cual establece un sistema nacional de aseguramiento de la calidad de la educación superior y que, entre otras acciones del Decreto № 239 del año 2016, plantea la necesidad de implementar mecanismos de diagnóstico para los estudiantes, que permitan identificar la brecha entre el perfil de ingreso real y el perfil de egreso por carrera y programa de pedagogía. No obstante, en lo que se refiere a la formación inicial docente (FID) en carreras de pedagogía, un foco problemático y relevante ha sido el cómo diagnosticar la brecha que existe entre el perfil de ingreso real y el perfil inicial ideal -implícito o explícito- de los estudiantes que ingresan a carreras universitarias que forman profesores (Ávalos, 2014).

Además, como requisito general para estos programas de formación y con especial atención a aquellos estudiantes que presenten mayores desafíos académicos, debe existir un plan de intervención que contemple estrategias de acompañamiento y nivelación. En esta línea, los diagnósticos se han enfocado principalmente en identificar los conocimientos previos (conocimientos declarativos) de los estudiantes que ingresan a la universidad (Stammen et al., 2018). Sin embargo, poco se ha realizado para intentar evaluar otras dimensiones importantes del perfil de ingreso de los estudiantes como las habilidades de razonamiento científico (Lawson, 2010; Villarroel y Bruna, 2014; Fisher et al., 2018) que traen consigo importantes beneficios. Por tanto, es relevante caracterizar en el perfil de ingreso, niveles de razonamiento científico de los estudiantes que ingresan a carreras de pedagogía para fortalecer, mejorar y/o ajustar estrategias de acompañamiento de la trayectoria académica.

La importancia del desarrollo del razonamiento está estrechamente relacionada con el "razonamiento operacional formal" (Piaget, 1970) y el "pensamiento crítico", por lo que el diagnóstico y desarrollo de habilidades de razonamiento científico también puede tener un impacto a largo plazo en el rendimiento académico de los estudiantes, (Coletta y Phillips, 2005). Por ejemplo, investigadores en el área de Física han utilizado el Test de Lawson (Bao et al., 2018), reconocido en áreas de ciencia, tecnología, ingeniería y matemáticas (STEM por sus siglas en inglés), encontrando correlaciones $(r \approx 0.5)$ entre las habilidades de razonamiento científico de los estudiantes y el aprendizaje de contenidos en Física. Atendiendo a estas consideraciones, desde el 2017 el Test de Lawson forma parte de una batería de instrumentos que componen un diagnóstico general inicial, aplicado a los estudiantes que ingresan a las carreras de pedagogía en una Universidad pública de Chile. Los resultados provenientes del diagnóstico podrán ser utilizados como insumos por las unidades académicas para fortalecer, mejorar y/o ajustar sus estrategias de acogida, nivelación y acompañamiento académico a estudiantes de primer año. Desde esta perspectiva, el Test de Lawson parece ser una ayuda significativa para los formadores de formadores, respecto a cómo orientar sus prácticas de enseñanza y aprendizaje para atender, de manera eficaz, la diversidad estudiantil.

Como objetivo principal, este estudio buscó determinar la relación entre el nivel de razonamiento científico y variables académicas de pre-ingreso de los estudiantes que ingresan a carreras de pedagogía, en el marco de la evaluación diagnóstica inicial, en una universidad pública chilena. Al respecto, las preguntas que orientaron esta investigación fueron: ¿Cuáles son los niveles de razonamiento científico en estudiantes que ingresan a carreras de pedagogía en primer año?, ¿Cómo se relaciona el nivel razonamiento científico (NRC) y variables académicas de pre-ingreso de los estudiantes que ingresan a carreras de pedagogía, en el marco de la evaluación nacional diagnóstica inicial en una universidad pública chilena?

\section{OTROS ANTECEDENTES}

Hay un par de aspectos que requieren ser analizados para el contexto de este trabajo: razonamiento científico y formación inicial de profesores, y niveles y habilidades de razonamiento científico

\section{Razonamiento científico y formación inicial de profesores}

Investigaciones recientes demuestran que el aprendizaje en el nivel universitario no depende exclusivamente de los conocimientos previos que los estudiantes poseen en su primer año de estudios, sino también de los niveles de desarrollo de sus habilidades de razonamiento científico (Fisher et al., 2018; Stammen et al., 2018).

En previos estudios, el razonamiento científico ha sido definido simplemente como pensar sobre algo con conocimiento científico (Zimmerman, 2007). No obstante, con base en las últimas investigaciones, se ha demostrado que el razonamiento científico depende no de una forma, sino de tres formas de conocimiento: i) conocimiento del contenido específico de conceptos de dominio apropiados, es decir, las entidades 
ontológicas que se utilizan para razonar. Por ejemplo, conceptos como átomo, gen o célula; ii) conocimiento procedimental, el cual es el conocimiento basado en los procesos utilizados para establecer afirmaciones sobre el saber. Por ejemplo, el concepto de variable, error, mediciones, causa y correlación, etc.; iii) conocimiento epistémico, el cual es el tipo de conocimiento asociado a las construcciones y valores epistémicos sobre cómo se utilizan argumentos para justificar las afirmaciones de la ciencia. Por ejemplo, teoría, hipótesis, lo que se constituye como evidencia, etc. (Osborne, 2018).

La investigación sobre el razonamiento científico y su importancia en el aprendizaje de los estudiantes se origina en los primeros estudios sobre las etapas del desarrollo cognitivo y el razonamiento formal (Piaget, 1970). Desde esta perspectiva psicológica, el autor plantea que el desarrollo cognitivo-intelectual de un sujeto ocurre en términos de cuatro etapas. Las dos primeras, se asocian al conocimiento procedimental y declarativo y son llamadas etapas de razonamiento sensorio-motor y pre-operacional, respectivamente. La tercera etapa, en cambio, se asocia a patrones de lógica en donde se aplica un razonamiento lógico para generar y/o describir objetos perceptibles; se manipulan variables empíricas observables; se generan y describen hipótesis causales; es decir, la etapa de razonamiento operacional concreto. Finalmente, la cuarta etapa se caracteriza por la adquisición del razonamiento lógico en todas las circunstancias, incluido el razonamiento abstracto; se asocia a la evaluación de hipótesis y/o relacionales causales y se denomina etapa de razonamiento operacional formal. En esta línea, los estudios de Lawson (2010) sobre razonamiento científico han considerado los aportes de Ausubel (1978) sobre los factores que influyen en el aprendizaje, como los "conocimientos" previos o sobre lo que el estudiante "ya sabe o conoce". Según Ausubel, el conocimiento previo de un alumno no es solo el conocimiento declarativo, sino que debe incluir el conocimiento descriptivo y el conocimiento proposicional o conocimiento de hechos. Por lo tanto, el "saber o conocer" requiere de la presencia de conocimientos procedimentales o habilidades de razonamiento como tal (Ausubel, 1978, p. 4).

Así, el razonamiento científico tiene una estructura que se desprende de la naturaleza hipotética-deductiva de la ciencia que incluye aspectos tales como el razonamiento proporcional, el control de variables, el razonamiento de probabilidad, el razonamiento de correlación y el proceso de extraer inferencias de premisas iniciales (Lawson, 2010). Desde esta definición, el razonamiento científico está asociado con el razonamiento inductivo y deductivo, vinculando la aplicación de los métodos de investigación científica a situaciones de razonamiento o resolución de problemas, como, por ejemplo, explorar sistemáticamente un problema, formular y probar hipótesis y evaluar resultados experimentales.

La capacidad de razonar científicamente ha sido considerada crucial para la formación de profesores a nivel mundial y a su vez se identifica como una habilidad necesaria para la "práctica" de la ciencia en varios países (Fisher et al., 2018). No obstante, dado que el razonamiento científico comprende habilidades cognitivas y procedimentales que son independientes del área de especialidad resulta también de interés estudiar cómo este se comporta en carreras de formación de profesores no científicas (Osborne, 2018). Específicamente, dado que se ha encontrado que el razonamiento científico es un predictor del rendimiento de los estudiantes en múltiples contextos, especialmente cuando se utilizan metodologías activas u orientadas a la investigación (Colleta et al., 2007; Lawson et al., 2007; Pérez de Landazábal et al., 2013).

Dada la importancia del razonamiento científico, diversas instituciones de educación e investigación científica, como la Asociación Internacional para la Evaluación del Logro Educativo (IEA), la Organización de las Naciones Unidas para la Educación, la Ciencia y la Cultura (UNESCO), la Asociación Americana para el Avance de la Ciencia (AAAS) y la Academia Nacional de Ciencias de Estados Unidos (NAS), han defendido, desarrollado y promovido estándares curriculares tanto a nivel nacional como internacional, posicionando al razonamiento científico como un tema universal (UNESCO, 2017). Este énfasis curricular, en el desarrollo de las habilidades de razonamiento de los estudiantes, implica que los futuros profesores también necesitan ser competentes en dichas habilidades para ayudar a sus estudiantes a desarrollar sus capacidades de razonamiento (Stammen et al., 2018). En Chile, si bien se han realizado estudios de medición de razonamiento científico en estudiantes universitarios (Pérez de Landazábal et al., 2013), no se han realizado estudios para determinar las habilidades de razonamiento científico de los profesores de secundaria (enseñanza media) ni tampoco de estudiantes de carreras de pedagogía que ingresan a primer año.

\section{Niveles y habilidades de razonamiento científico}

Se han desarrollado varios instrumentos para evaluar el razonamiento científico de los estudiantes; sin embargo, gran parte de ellos han tendido a centrarse en contextos más amplios, como alfabetización científica, y ética en entornos socioculturales (Ding et al., 2016). Por ello, para medir las sub-habilidades de razonamiento científico y el NRC, este estudio emplea uno de los instrumentos más utilizados en la literatura, el Test de Lawson (Lawson, 2010). La prueba no pretende que se aplique el conocimiento, sino que se razone. Por ello, la respuesta y su justificación deben basarse en la información que se presenta en el propio texto y no se necesita acudir a conocimientos previos (Pérez et. al., 2013). Por estas consideraciones, este Test ha ganado gran popularidad en la comunidad educativa STEM (Bao et. al., 2018). El Test de Lawson fue diseñado para evaluar la capacidad de razonamiento científico, de acuerdo con las propuestas de Piaget. La 
prueba evalúa cinco aspectos del razonamiento científico y con sus respectivas sub-habilidades (Lawson, 2010), como se muestran en la Tabla 1. Estos aspectos se evalúan de manera aislada o combinada entre las 24 preguntas de la prueba (Rodríguez et al., 2010).

Tabla 1: Distribución de ítems según ejes temáticos y descripción de Sub-Habilidades de Razonamiento Científico

\begin{tabular}{|l|l|c|}
\hline Eje temático & Descripción de sub-habilidades & № de ítems \\
\hline $\begin{array}{l}\text { Conservación de } \\
\text { magnitudes físicas }\end{array}$ & $\begin{array}{l}\text { El estudiante aplica el razonamiento de conservación de objetos } \\
\text { perceptibles y sus propiedades. }\end{array}$ & 4 \\
\hline $\begin{array}{l}\text { Pensamiento de } \\
\text { proporcionalidad }\end{array}$ & $\begin{array}{l}\text { El estudiante reconoce e interpreta las relaciones descritas por variables } \\
\text { observables. }\end{array}$ & 4 \\
\hline $\begin{array}{l}\text { Identificación y control } \\
\text { de variables }\end{array}$ & $\begin{array}{l}\text { El estudiante reconoce la necesidad de considerar todas las posibles } \\
\text { variables causales y diseñar un experimento en el que controlen todas las } \\
\text { variables excepto la que está siendo investigada. }\end{array}$ & 6 \\
\hline $\begin{array}{l}\text { Pensamiento } \\
\text { probabilístico }\end{array}$ & $\begin{array}{l}\text { El estudiante reconoce fenómenos de carácter probabilístico y evalúa la } \\
\text { probabilidad de que ciertas suposiciones se mantengan verdaderas en el } \\
\text { diseño de un experimento. }\end{array}$ & 4 \\
\hline $\begin{array}{l}\text { Pensamiento } \\
\text { combinatorio y } \\
\text { correlacional }\end{array}$ & $\begin{array}{l}\text { El estudiante considera las combinaciones posibles de condiciones } \\
\text { experimentales, aunque algunas no se realicen en la naturaleza; y a pesar } \\
\text { de fluctuaciones aleatorias, el estudiante reconoce causas o relaciones de } \\
\text { un fenómeno en estudio. }\end{array}$ & 6 \\
\hline
\end{tabular}

Este Test ha sido validado para su uso en el aula (Coletta y Phillips, 2005) y su utilización permite una rápida y eficiente comparación con otras muestras. Para este estudio, el Test tomó valores porcentuales entre 0 y 100 que representan el porcentaje de aciertos del estudiante a las 24 preguntas pareadas que tiene el instrumento. Dependiendo del número de pares correctos que obtengan en esta prueba, se consideran tres niveles de razonamiento científico: concreto, transicional y formal y las sub-habilidades que cada uno de ellos lo caracteriza.

Razonamiento científico concreto (puntaje igual o inferior a 33.3\%, 0 a 4 pares correctos): Los estudiantes de este nivel poseen habilidades para manipular variables empíricas observables, realizar descripciones de fenómenos naturales y para categorizar objetos, eventos y situaciones. A su vez, son capaces de: a) comprender enunciados o términos que hagan referencia a situaciones familiares directas y objetos observables, en términos de simples asociaciones; b) seguir instrucciones paso a paso siempre que cada paso esté completamente especificado, y c) relacionar su punto de vista con el de otro en situaciones conocidas.

Razonamiento científico transicional (puntaje mayor a 33.3\% y menor a 75,0 \%, 5 a 8 pares correctos): Los estudiantes de este nivel tienen habilidades para integrar elementos y características tanto del nivel formal como del nivel concreto. Los estudiantes que se encuentran en este nivel podrían ser capaces de: a) probar hipótesis causales que involucren agentes visibles y que correspondan con la variable independiente de un experimento; b) buscar e identificar algunas variables causales, pero no de manera sistemática; c) hacer observaciones y generar hipótesis. Sin embargo, en contextos causales no logran probar dichas hipótesis, presentando dificultades al momento de: d) iniciar su razonamiento desde lo hipotético; e) responder a problemas complejos aplicando una regla correcta (sí aplican reglas relacionadas) y f) procesar información siendo consciente de su propio razonamiento. Por último, son capaces de establecer asociación entre variables, siempre y cuando estas no sean abstractas. Un estudiante está en el nivel más alto de la escala ordinal de razonamiento cuando consistentemente demuestra tener un razonamiento formal (Ates y Cataloglu, 2007).

Razonamiento científico formal (puntaje superior a $75.00 \%$, entre 8 y 12 pares correctos): Los estudiantes de este nivel tienen habilidades que les permiten: probar hipótesis causales que involucran agentes invisibles 0 abstractos en alguna de las variables de un experimento; usar hipótesis causales como punto de partida de su razonamiento y probarlas mediante razonamiento hipotético-predictivo, además, los estudiantes pueden imaginar posibles factores causales, deducir las consecuencias de ciertas posibilidades y luego verificar empíricamente qué consecuencias tienen estas.

\section{METODOLOGÍA}

La investigación se enmarca en un estudio transeccional, correlacional-causal, de acuerdo con la tipología propuesta por Hernández et. al., (2014). Los instrumentos utilizados para la recolección de datos fueron la aplicación del Test de Lawson y la información recopilada sobre bases de datos preexistentes respecto al perfil de ingreso de los estudiantes proporcionados por la universidad. El test de Lawson se utiliza para evaluar la capacidad de razonamiento científico de acuerdo con las propuestas de Piaget. Este instrumento se ha utilizado durante casi tres décadas y representa una evaluación basada en tareas como la formulación y experimentación de hipótesis, y afirma medir el razonamiento científico (Bao et al., 2018). En el contexto de este estudio, los datos académicos sobre variables de pre-ingreso fueron proporcionados por la Vicerrectoría 
Académica de la universidad. Cabe señalar que, en lo formal y ético, la recolección de datos cumplió con todos los estándares y protocolos institucionales en coherencia con el comité de ética de la universidad. Una de las limitaciones metodológicas del estudio hace referencia a que la prueba es opcional para las carreras que forman profesores, lo que podría afectar al seguimiento y acompañamiento de los estudiantes año a año entre diferentes cohortes.

\section{Instrumento}

El instrumento utilizado corresponde a la versión traducida al español por Pérez de Landazábal et al. (2005) del test de Lawson en su versión del año 2010, mostrando una alta confiabilidad (alfa de Cronbach de 0.78). En este estudio, el test fue adaptado al contexto nacional y aplicado por primera vez en el año 2017, a 209 estudiantes de la misma institución de educación superior alcanzando una confiabilidad de 0.79 . No obstante, para el año 2018, se realizaron mejoras asociadas principalmente a la redacción y numeración de algunos ítems, manteniendo una alfa de Cronbach de 0.79. En este estudio, fueron evaluadas las habilidades de razonamiento científico de 158 estudiantes pertenecientes a cinco carreras de pedagogía de una Universidad pública en Chile: dos carreras de pedagogía no científicas (C1 y C2) y tres carreras de pedagogía en ciencias (C3, C4 y C5). Siendo una de ellas (C5), un Plan de Prosecución de Estudios para profesores que se encuentren haciendo clases en Establecimientos de Enseñanza Media o Básica por lo menos con un año de antelación al ingreso al programa en posesión de un título profesional afín a la carrera de pedagogía a la cual ingresaron.

Si bien, el diagnóstico en un comienzo solo consideraba carreras de pedagogías científicas, se considera C1 y C2 dado que, por un lado, C1 tiene como requisito la Prueba de Selección Universitaria (PSU) de Ciencias, la cual tiene un $20 \%$ de ponderación en el puntaje de ingreso requerido por la carrera; además, el perfil de los estudiantes posee un sello curricular distintivo y único, vinculado a Filosofía de las Ciencias. Por otro lado, C2, tiene un proyecto educacional que apunta al desarrollo de una dimensión cognitiva que incluye el dominio de la especialidad junto con el desarrollo de una capacidad creadora, una actitud crítico-investigativa. Esta decisión metodológica se tomó, por un lado, considerando la importancia de la alfabetización científica como eje transversal en la formación docente, donde más allá de la capacidad de referir temas de ciencias, es necesario consolidar -sobre todo en los docentes- la comprensión o el significado del lenguaje científico en sus múltiples disciplinas y en diversos contextos de uso (Sjöström y Eilks, 2018). Por otro lado, la decisión se sustenta en el valor y en la necesidad de desarrollar un pensamiento crítico en los docentes, con miras al posicionamiento social, político y educativo. Bajo estas premisas, el razonamiento científico trasciende a ser netamente una cuestión de carreras que forman profesores en el área de ciencias.

\section{Variables y modelos de análisis}

El estudio consideró las siguientes variables: i) variables nominales cualitativas: sexo, comuna, dependencia centro escolar (municipal, particular-subvencionado, particular-pagado), carrera de pedagogía a la cual ingresó el estudiante, nivel de razonamiento científico y vía de acceso a la universidad; ii) variables cuantitativas: edad, puntaje en Prueba de Selección Universitaria (PSU) de Ciencias, Lenguaje, Matemática e Historia, puntaje Ranking de Notas (este puntaje expresa la posición relativa del estudiante en cada contexto educativo en el cual estuvo durante su Enseñanza Media, toma como referencia el desempeño académico de las últimas tres generaciones y puntaje Notas de Enseñanza Media (NEM).

Para el caso de la variable vía de acceso, es importante destacar que la Universidad donde se realizó el estudio tiene diferentes vías de acceso a las carreras de pedagogías, tales como: Cupo talento científico (cupo para jóvenes que poseen una trayectoria científica destacada), Cupo capacidades diferentes (cupo que busca promover el ingreso de personas en situación de discapacidad, Programa Educadores Líderes (cupo para estudiantes de todo el país, que estén pasando a tercer año de educación media y que manifiestan inquietud por las pedagogías a temprana edad, Cupo Pueblos Indígenas, Cupo Deportista Destacado, Cupo Hijo de Funcionario, Ingreso Extranjero, Cupo PACE (Programa de acceso y de acompañamiento a la educación superior). Este último, acompaña a los estudiantes mediante acciones de preparación y apoyo permanentes, buscando asegurar cupos adicionales a la oferta académica regular por parte de las IES participantes y a la vez, facilitando el progreso de los estudiantes, a través de mecanismos de acompañamiento previos y durante la trayectoria académica. A su vez, busca restituir el derecho a la educación superior, garantizando un cupo a estudiantes de sectores vulnerados que acceden sin los puntajes PSU regulares. Para el análisis estadístico con las variables descritas anteriormente, se diseñaron modelos de regresión multi-variable con el propósito de establecer correlaciones y ver la incidencia de las variables mencionadas anteriormente en el NRC de los estudiantes. En el análisis de datos y el diseño de dichos modelos se empleó el software STATA v.15.1.

No obstante, se decidió no considerar aquellas variables con poca o nula incidencia en el NRC como edad, comuna y dependencia de centro escolar. Los modelos diseñados para el estudio fueron los siguientes: i) Modelo 1, incluyó la variable sexo y variable puntaje obtenido en Pruebas de Selección Universitaria de Ciencias y Lenguaje (se han omitido las otras pruebas dado valor de $\mathrm{P}>0.05$ ) (ver resultados); ii) Modelo 2 , 
incluyó las variables puntaje NEM y puntaje Ranking, dada la correlación de la variable con NRC y iii) Modelo 3 , incluyó las variables vía de acceso a las pedagogías, en especial vía 4: PACE. Se omitieron otras vías de ingreso por ser variables colineales que no influyen en los valores de $P$.

\section{RESULTADOS}

Los resultados presentados buscan, primeramente, describir los niveles de razonamiento científico de 158 estudiantes. Para esto se presentan variables estadísticas descriptivas generales y luego, se caracterizan los niveles y sub-habilidades de razonamiento científico de los estudiantes. A continuación, se relacionan con variables académicas de pre-ingreso y se presentan tres modelos que contribuyen a identificar el comportamiento de la variable Nivel de Razonamiento Científico de estudiantes que ingresan a carreras de pedagogía.

\section{Niveles de razonamiento científico de la muestra}

A partir de la información, se puede observar que del total de los estudiantes que respondieron el Test $41.77 \%$ (66 estudiantes) se encuentra en un nivel de razonamiento concreto; $52.53 \%$ (83 estudiantes) está en un nivel de razonamiento científico transicional y finalmente, un $5.70 \%$ (9 estudiantes) alcanzan el nivel de razonamiento formal. En cuanto a la variable sexo, el $48.86 \%$ de las mujeres presentaron niveles de razonamiento concreto, $47.73 \%$ alcanzan el nivel de razonamiento científico transicional y $3.41 \%$ obtienen el nivel de razonamiento formal. Estos resultados tienen un coeficiente de Pearson $x^{2}=5.0881$ y un $\operatorname{Pr}=0.079$, lo que se traduce, por ahora, en una correlación débil entre estas dos variables. Los descriptores estadísticos generales fueron: Valor mínimo: 1, Valor máximo: 11, Promedio: 5, Desviación estándar: 2.13. Los resultados presentados en la Tabla 2 tienen un coeficiente de Pearson chi $^{2}=5.0881$ y un $\operatorname{Pr}=0.079$.

Tabla 2: Nivel de Razonamiento Científico a Nivel General y Según Sexo

\begin{tabular}{|l|l|l|l|}
\hline & \multicolumn{2}{|c|}{ Sexo } & \\
\hline Nivel de razonamiento & Masculino & Femenino & Total \\
\hline Concreto & 23 & 43 & 66 \\
\hline$\%$ & 32.86 & 48.86 & 41.77 \\
\hline Transicional & 41 & 42 & 83 \\
\hline$\%$ & 58.57 & 47.73 & 52.53 \\
\hline Formal & 6 & 3 & 9 \\
\hline$\%$ & 8.57 & 3.41 & 5.70 \\
\hline Total & 70 & 88 & 158 \\
\hline
\end{tabular}

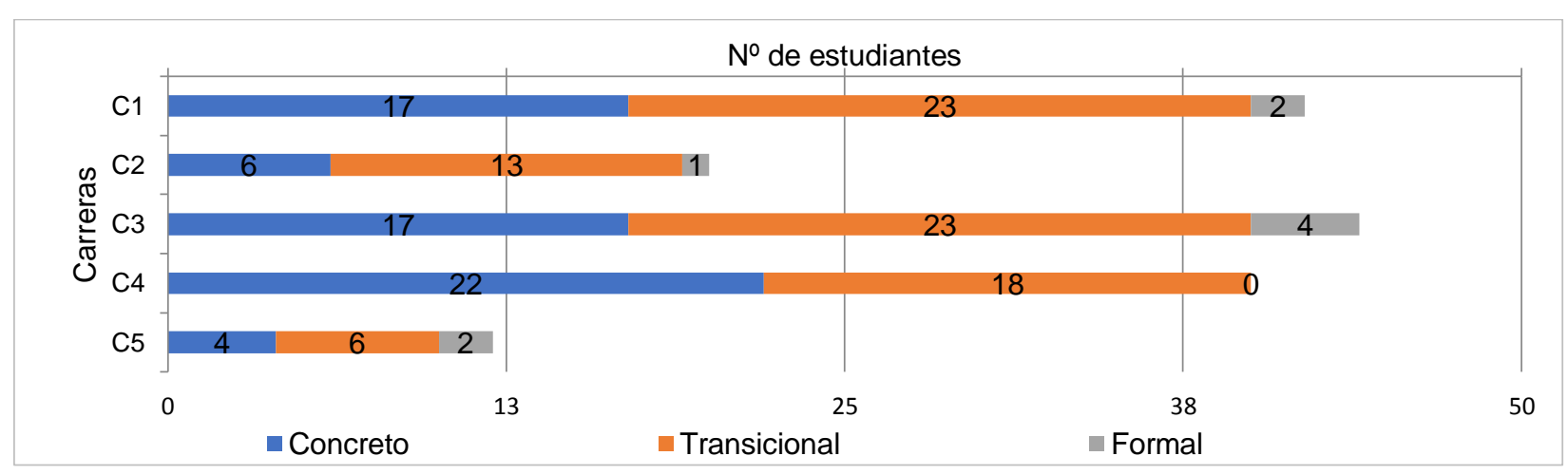

Fig. 1. Distribución del Niveles de Razonamiento Científico y frecuencia de estudiantes por Carrera

\section{Nivel de razonamiento científico por carrera}

En la Figura 1, se identifican los niveles de razonamiento científico en cada una de las carreras que componen el estudio. A nivel general se puede apreciar que las carreras muestran una distribución homogénea en los niveles de razonamiento transicional (entre 50 y $65 \%$ de los estudiantes), (ver detalles en Tabla 3 ). Sin embargo, C4 alcanza el mayor número de estudiantes en un nivel concreto, dado que es una carrera con alta exigencia en puntaje PSU. En contraste, y siendo una carrera no-científica, C2 alcanza el nivel más bajo de estudiantes en este nivel. Por otro lado, C5 muestra tener los niveles más altos de nivel de razonamiento transicional-formal dado que es una carrera de regularización de estudios.

\section{Sub-habilidad de razonamiento científico por carrera}

La identificación de las sub-habilidades que componen el razonamiento científico, forman parte de la caracterización de los niveles de razonamiento. En los resultados presentados en la Tabla 4 ( $\Delta \%$ representa la diferencia entre cada carrera y el \% promedio de aprobación del total de estudiantes de la Universidad) y 
la Figura 2, podemos apreciar el detalle por sub-habilidades de razonamiento científico. A nivel general, se observa que las habilidades con mayor porcentaje de aprobación fueron conservación de magnitudes físicas y pensamiento probabilístico. En contraste y como claramente ilustra la Figura 2, las sub-habilidades de razonamiento científico con menor logro fueron pensamiento de proporcionalidad e identificación y control de variables. Dichos resultados serán discutidos en la siguiente sección. En la Figura 2, C1 y C2, representan carreras no científicas, C3, C4, C5 representan carreras científicas con C5 siendo un programa de prosecución de estudios en pedagogía. Finalmente representa el promedio de aprobación de todas las carreras.

Tabla 3: Nivel de Razonamiento Científico alcanzado por Estudiantes según Carrera de Pedagogía.

\begin{tabular}{|l|l|l|l|l|l|l|l|}
\hline & \multicolumn{3}{|l|}{ Nivel de Razonamiento Científico } & & \\
\hline Carrera & Concreto & $\%$ & Transicional & $\%$ & Formal & $\%$ & Total \\
\hline C1 & 17 & 40 & 23 & 55 & 2 & 5 & 42 \\
\hline C2 & 6 & 30 & 13 & 65 & 1 & 5 & 20 \\
\hline C3 & 17 & 39 & 23 & 52 & 4 & 9 & 44 \\
\hline C4 & 22 & 55 & 18 & 47 & 0 & 0 & 40 \\
\hline C5 & 4 & 33 & 6 & 50 & 2 & 17 & 12 \\
\hline Total & 66 & 41.77 & 83 & 52.53 & 9 & 5.70 & 158 \\
\hline
\end{tabular}

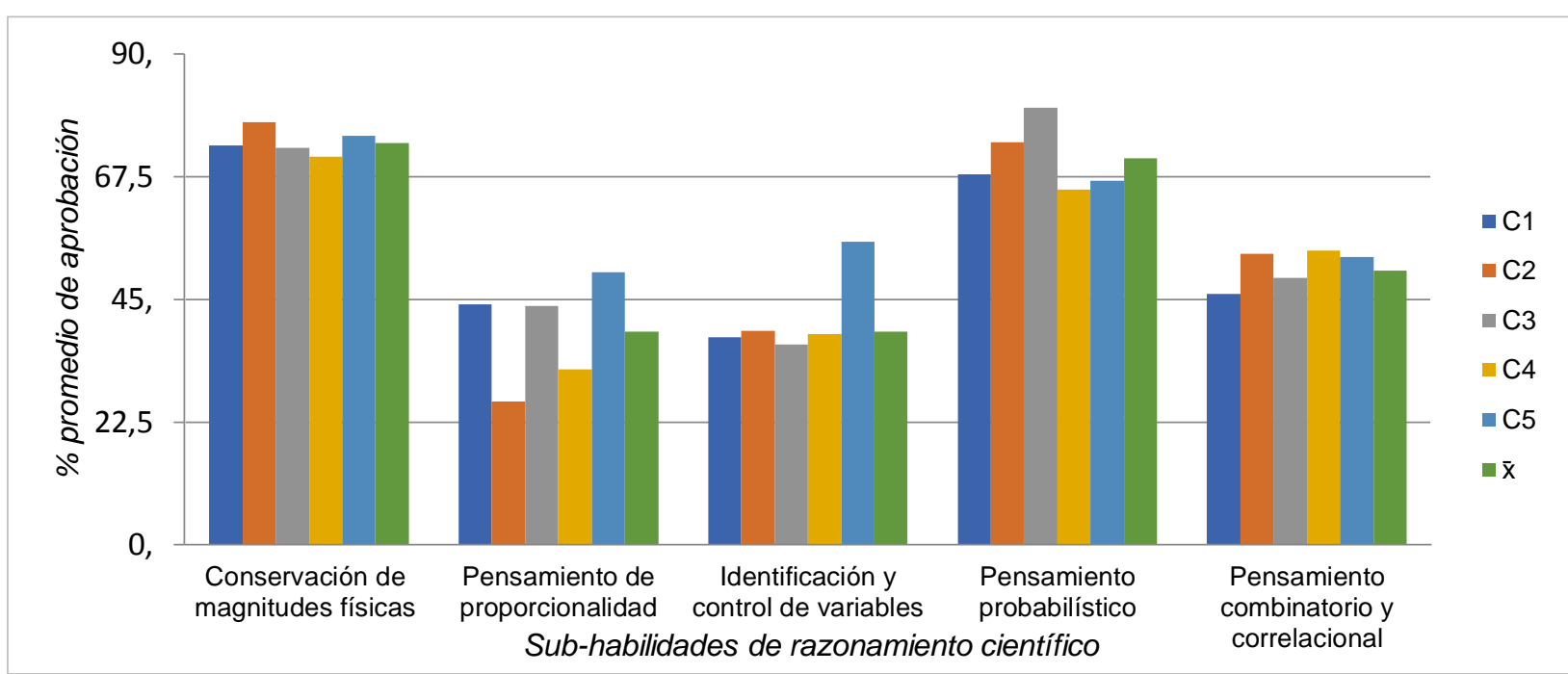

Fig. 2: Comparación \% de logro por sub-habilidades de razonamiento científico.

\section{Modelos de correlación lineal multi-variable}

Con el propósito de responder a la segunda pregunta de investigación, se procedió a modelar la variable número de pares correctos y su relación con variables académicas categóricas de pre-ingreso. Para ello se crearon diferentes modelos para analizar qué variables estarían afectando el número de pares correctos que alcanzan los estudiantes. Como se mencionó en la metodología, las variables comuna, dependencia y edad, fueron eliminadas por tener baja incidencia.

Tabla 4: Porcentaje de logro Según Sub-Habilidades de Razonamiento Científico por Carrera

\begin{tabular}{|c|c|c|c|c|c|c|c|c|c|c|c|}
\hline $\begin{array}{l}\text { Sub-habilidad de } \\
\text { razonamiento científico }\end{array}$ & $\begin{array}{l}\text { \% promedio } \\
\text { aprobación } \\
\text { Universidad }\end{array}$ & $\begin{array}{l}\mathrm{C} 1 \\
\mathrm{n}=42\end{array}$ & $\begin{array}{l}\Delta \\
\%\end{array}$ & $\begin{array}{l}\mathrm{C} 2 \\
\mathrm{n}=20\end{array}$ & $\Delta \%$ & $\begin{array}{l}\mathrm{C} 3 \\
\mathrm{n}=44\end{array}$ & $\begin{array}{l}\Delta \\
\%\end{array}$ & $\begin{array}{l}\mathrm{C} 4 \\
\mathrm{n}=38\end{array}$ & $\Delta \%$ & $\begin{array}{l}\mathrm{C} 5 \\
\mathrm{n}=12\end{array}$ & $\Delta \%$ \\
\hline $\begin{array}{l}\text { Conservación de } \\
\text { magnitudes físicas }\end{array}$ & 73.6 & 73.2 & 0.4 & 77.5 & 3.9 & 72.7 & 0.8 & 71.1 & 3.0 & 75 & 1.4 \\
\hline $\begin{array}{l}\text { Pensamiento de } \\
\text { proporcionalidad }\end{array}$ & 39.1 & 44 & 5 & 26.3 & $\begin{array}{l}12 . \\
8\end{array}$ & 43.8 & 4.7 & 32.2 & $\begin{array}{l}12 . \\
8\end{array}$ & 50 & $\begin{array}{l}10 . \\
9\end{array}$ \\
\hline $\begin{array}{l}\text { Identificación y control de } \\
\text { variables }\end{array}$ & 39.1 & 38.1 & 1 & 39.2 & 0 & 36.7 & 2.4 & 38.6 & 0 & 55.6 & $\begin{array}{l}16 . \\
4\end{array}$ \\
\hline Pensamiento probabilístico & 70.9 & 67.9 & 3 & 73.8 & 2.9 & 80.1 & 9.2 & 65.1 & 2.9 & 66.7 & 4.2 \\
\hline $\begin{array}{l}\text { Pensamiento combinatorio } \\
\text { y correlacional }\end{array}$ & 50.3 & 46 & 4.3 & 53.3 & 3.0 & 48.9 & 1.5 & 53.9 & 3.0 & 52.8 & 2.5 \\
\hline
\end{tabular}


En resumen, de los resultados obtenidos de la Tabla 5, se puede apreciar que la variable sexo (en específico femenino) al estar incluida en el modelo 3 , estaría afectando el nivel de razonamiento $(P=0.045)$. Lo mismo ocurre con la vía de ingreso 4 (PACE) con un $P=0.014$. Además, se observa que las pruebas de selección universitaria (PSU) en especial Ciencias y Lenguaje tienen una fuerte relación con el nivel de razonamiento científico alcanzado por los estudiantes con valores $P=0.01$ y $P<10^{-3}$, respectivamente. También, se puede apreciar cómo varían los valores estadísticos significativos de $P$ al ir agregando variables a los modelos. Para lo cual, se ha aplicado prueba VIF para evaluar significancia de variables. La prueba VIF se aplica luego de hacer pruebas de correlación y sive para conocer la multicolinealidad de variables en los modelos multivariables. Significa que la variable podría considerarse como una combinación lineal de otras variables independientes. Además, para complementar los resultados obtenidos, se presentan las relaciones entre los puntajes obtenidos por los estudiantes en cada una de las pruebas universitarias y su relación con el número de pares correctos alcanzados en el Test de Lawson. En la Figura 3, se ilustran las regresiones obtenidas con el software STATA, con el cual se generaron los gráficos y incidencia entre puntaje PSU y NRC.

Tabla 5: Modelos de Regresión Lineal Multi-Variable para la Predicción del Nivel de Razonamiento Científico (NRC)

\begin{tabular}{|c|c|c|c|c|c|c|}
\hline & & \multicolumn{5}{|l|}{ Modelo } \\
\hline Variables & & 1 & & 2 & & 3 \\
\hline $\mathrm{R}$ & & 0.625 & & 0.639 & & 0.67 \\
\hline $\mathrm{R}^{2}$ & & 0.392 & & 0.408 & & 0.4505 \\
\hline $\mathrm{R}^{2}$ ajustado & & 0.375 & & 0.379 & & 0.4005 \\
\hline$P$ para $\mathrm{R}^{2}$ ajustado & & - & & 0.005 & & 0.026 \\
\hline & $\beta(95 \% \mathrm{Cl})$ & $P$ & $\beta(95 \% \mathrm{Cl})$ & $P$ & $\beta(95 \% \mathrm{Cl})$ & $P$ \\
\hline Sexo & $-0.624(-1.269-0.018)$ & 0.057 & $-0.623(-1.26-0.016)$ & 0.056 & $-0.667(-1.320-0.0141)$ & 0.045 \\
\hline Puntaje PSU Ciencias & $0.007(0.002-0.012)$ & 0.008 & $0.006(0.001-0.012)$ & 0.018 & $0.007(0.001-.0133)$ & 0.010 \\
\hline $\begin{array}{l}\text { Puntaje PSU } \\
\text { Lenguaje }\end{array}$ & $0.011(0.062-0.0170$ & $P<10^{-3}$ & $0.011(0.055-0.016)$ & $P<10^{-3}$ & $0.012(0.007-0.0182)$ & $P<10^{-3}$ \\
\hline Puntaje NEM & & - & $1.160(-1.39-3.718)$ & 0.370 & $0.907(-1.752-3.56)$ & 0.500 \\
\hline Puntaje Ranking & & - & $-0.006(-0.014-0.002)$ & 0.143 & $-0.003(-0.012-0.005)$ & 0.472 \\
\hline Vía de ingreso $4^{*}$ & & - & & & $\begin{array}{l}1.973(0.402- \\
3.544)\end{array}$ & 0.014 \\
\hline
\end{tabular}

\section{DISCUSIÓN}

A partir de los resultados, podemos observar que la mayoría de los estudiantes se ubican en los niveles de razonamiento concreto $(41,77 \%)$ y razonamiento transicional $(52,53 \%)$. A su vez, llama la atención que solo un $5,7 \%$ de los estudiantes se ubican en el nivel de razonamiento formal. Dichos hallazgos, coinciden con diagnósticos internacionales en donde los estudiantes mostraron niveles de razonamiento similares a este estudio (Pérez et, al., 2013). Estos resultados, podrían estar indicando que un bajo porcentaje de estudiantes logra evaluar y/o relacionar hipótesis causales que involucran agentes invisibles o abstractos en alguna de las variables de un experimento o utilizar hipótesis causales como punto de partida mediante un razonamiento hipotético-predictivo (Lawson, 2010).

Dicha dificultad de los estudiantes en estas habilidades podría atribuirse al poco énfasis que los docentes asignan al desarrollo de experiencias y/o prácticas de laboratorio. Por lo cual, concretamente, se sugiere articular los métodos tradicionales de enseñanza con metodologías activas y basadas en: argumentación, indagación e investigación experimental (Fisher et, al., 2018; Weld et al., 2011; Zimmerman, 2007; Lobato y Madinabeitia, 2011). Además, se sugiere el uso de experiencias de laboratorio en donde los estudiantes puedan utilizar simuladores y softwares para modelar variables (Liao y She, 2009; Gunhaart y Srisawasdi, 2012). De esta manera, se estará fomentando la capacidad de razonar de los estudiantes como eje fundamental en su trayectoria académica y como parte de su alfabetización científica.

Concretamente, para fortalecer el NRC de los estudiantes que se ubican en el nivel de razonamiento concreto una posible vía es el diseño o adaptación de actividades de aprendizaje para estudiantes de primer año, que incluyan objetos tangibles y observables para consolidar las habilidades de pensamiento asociadas a ese nivel (Coletta et al., 2007). Junto con esto, se sugiere revisar las secuencias de contenidos de los primeros cursos, más aún cuando se introducen por primera vez conceptos que involucran un alto nivel de razonamiento y abstracción. En este sentido, la literatura plantea que, los programas de asignaturas introductorias deben ajustarse a partir de la enseñanza de contenidos concretos, e ir acompañando el proceso de enseñanza-aprendizaje-evaluación con estrategias explícitas que aborden habilidades de razonamiento científico lo cual, ha demostrado conducir a un mejor desempeño en contenidos teóricos más abstractos y complejos (Moore, 2019). En segundo lugar, los estudiantes que se ubican en nivel de razonamiento transicional se verán favorecidos con una inmersión explícita en actividades orientadas a abordar el razonamiento científico. Por ejemplo, haciendo explícita la enseñanza de habilidades razonamiento de tipo hipotético-deductivo por medio de actividades experimentales (Lawson, 2010). Este enfoque explícito para el 
desarrollo de razonamiento parece dar como resultado un aumento en el NRC, en donde los profesores encargados de los procesos de formación proveen a los futuros maestros la habilidad de distinguir entre la respuesta dan a un problema y los procedimientos o estrategias que emplean en dicho proceso (Cerda et al., 2017). Finalmente, se sugiere que los estudiantes que se ubican en el nivel de razonamiento formal participen colaborativamente en desarrollar estrategias de andamiaje con sus pares de niveles más bajos. Así, podrían contribuir a consolidar estas habilidades, aprovechando la zona de desarrollo próximo, mientras refuerzan y consolidan las propias (Labarrere, 2016).
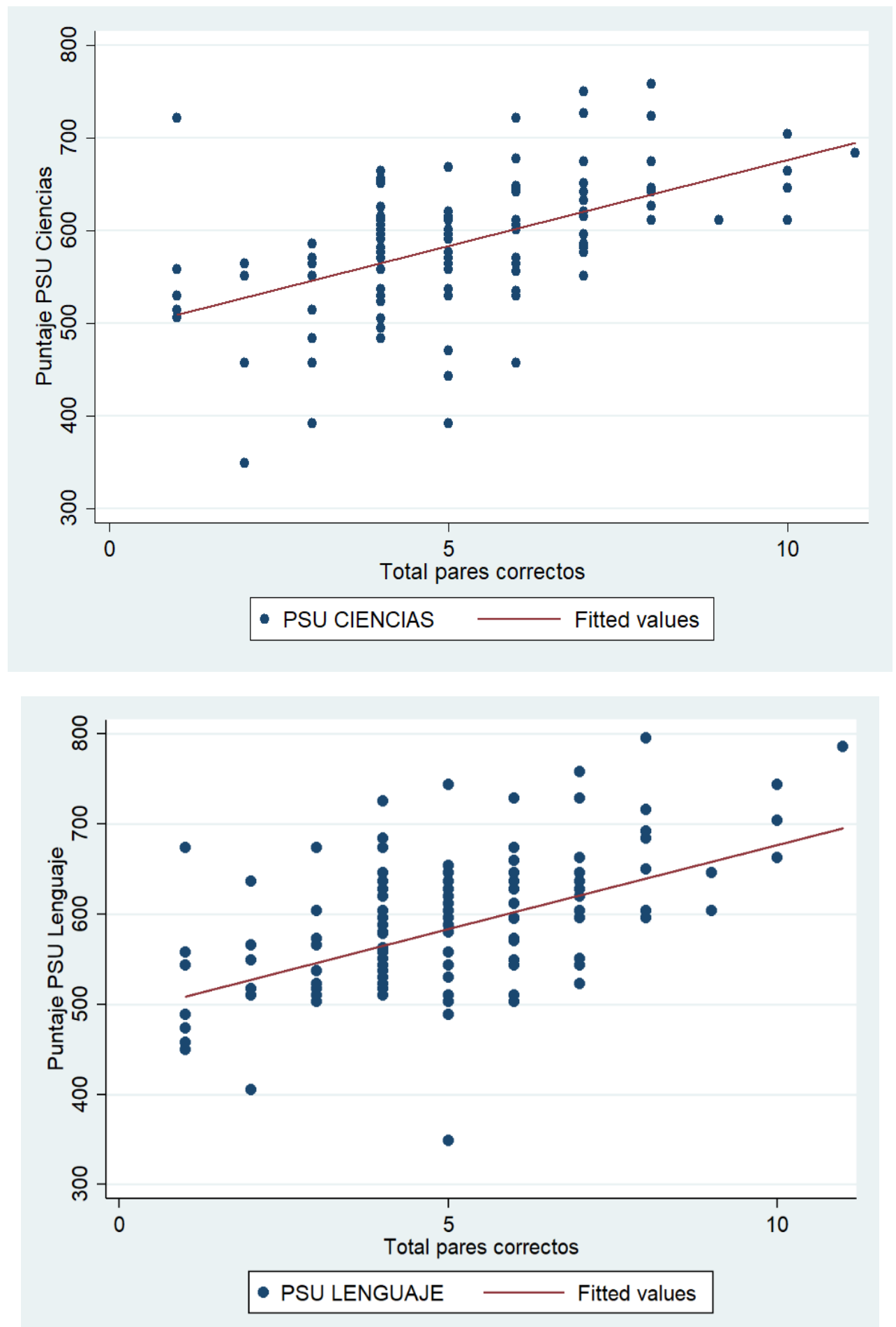

Fig. 3. Regresión lineal puntajes PSU y relación con la cantidad de pares correctos en el Test de Lawson.

De manera específica, el estudio mostró que solo un 39.1\% de los estudiantes logra reconocer e interpretar las relaciones descritas por variables observables y/o logran reconocer fenómenos de carácter probabilístico, al evaluar la probabilidad de que ciertas suposiciones se mantengan verdaderas en el diseño de un experimento (conservación de magnitudes físicas y pensamiento probabilístico). Los bajos resultados, se 
acentuaron más en las carreras no científicas ( $\mathrm{C} 1$ y $\mathrm{C} 2$ ) con solo un $26.3 \%$ y $32.2 \%$ de aprobación en subhabilidades de razonamiento asociadas al pensamiento proporcional y probabilístico. Esto podría atribuirse a un bajo desarrollo de competencias matemáticas (Piraksaa et al., 2014). No obstante, es importante destacar que dichos niveles tampoco fueron significativamente altos para carreras científicas. Esto se podría indagar con mayor profundidad en un nuevo estudio, considerando investigaciones actuales sobre el rol del conocimiento específico y general para el desarrollo del razonamiento científico (Fisher et, al., 2018).

Por otro lado, a partir de los modelos de regresión multi-variable, las Pruebas de Selección Universitaria de Ciencias y Lenguaje mostraron una alta correlación con el total de pares correctos $\left(P<10^{-3}\right.$ y $\left.P=0.008\right)$. Esto quiere decir que a mayor puntaje en las pruebas de selección un estudiante tendrá mayor número de pares correctos en el Test de Lawson. Sin embargo, dado que muchas IES optan por mecanismos basados en la diversificación de las vías de acceso (Zárate-Rueda et al., 2017) se hace necesario, tomar acciones concretas para acompañar a los estudiantes que ingresan a las carreras de pedagogía con bajos puntajes PSU y que contribuyan a fortalecer el nivel de razonamiento científico. Respecto a las estrategias de acción a promover, se sugiere evaluar distintas alternativas de apoyo y derivación de los estudiantes, las cuales deben discutirse y analizarse al interior de cada comité de carrera independiente de que la modalidad de apoyo sea transversal o focalizada. La idea es que las estrategias que desarrolle la unidad se complementen con el trabajo de programas de apoyo al estudiante de cada facultad, para evitar duplicidades y optimizar el seguimiento conjunto de los resultados académicos de los estudiantes. Al respecto, es posible utilizar cursos o cápsulas on-line para abordar la nivelación, reforzamiento de determinadas materias, articulación con asignaturas que trabajan o requieren de las habilidades y conocimientos reportados.

Finalmente, otras variables de ingreso como NEM (Notas de Enseñanza Media) y Ranking, no fueron significativas como muestra el modelo 2 de la Tabla 5. Sin embargo, el valor $\mathrm{P}=0.045$ de la variable sexo se hace significativo al introducir otras variables al modelo 3 , en específico vía de ingreso. Si bien existen investigaciones que indican que no hay una diferencia significativa en el razonamiento científico respecto a la variable sexo (Al-Zoubi et al., 2009; Piraksaa et al., 2014), sería interesante indagar cómo esto sumado a otras variables de pre-ingreso puede cambiar. Lo anterior, dado que, según este estudio y de acuerdo con el modelo 3, dicha brecha de sexo se acentúa para estudiantes de la vía de acceso 4, que corresponde a estudiantes que ingresan vía el Programa de acceso y de acompañamiento a la educación superior (PACE).

\section{CONCLUSIONES}

A partir de los resultados hallados y de su discusión es posible dar respuesta a las preguntas de investigación y concluir que: (1) Los niveles de razonamiento científico de los estudiantes que ingresan a carreras de pedagogía de primer año se ubican principalmente en niveles transicionales; (2) dos de las sub-habilidades evaluadas en las carreras no científicas, obtienen porcentajes bajos en "pensamiento proporcional e identificación y control de variables, estos dos aspectos cuestionan por nuevas estrategias de enseñanza y aprendizaje que promuevan niveles formales de razonamiento: (3) A través de los modelos de correlación se constata que existe una relación entre el NRC y la variable cualitativa "sexo". Específicamente, la subcategoría "femenino". A su vez, las variables académicas de pre-ingreso, como el puntaje obtenido en la prueba de selección universitaria (PSU) en ciencias y lenguaje, se correlacionan positivamente con el NRC. Lo anterior, se acentúa para estudiantes que han accedido mediante el programa PACE. Este conjunto de información exige pensar en opciones adecuadas para fomentar las habilidades de razonamiento científico que sugieren algunas acciones como: a) planificación de los contenidos en forma ascendente en la demanda cognitiva (concreto a lo abstracto); b) apoyo a estudiantes con dificultades académicas que incluyan aspectos disciplinares, procedimentales y actitudinales; c) diseño y evaluación de estrategias didácticas con un predominio de aspectos metacognitivos como la argumentación, la explicación, la lectura académica y, d) enfoques de enseñanza alternativos como indagación, investigación, aprendizaje basado en problemas, entre otros: y (4) Si bien estos son resultados para un contexto específico, es recurrente que las Instituciones de Educación Superior analizan otras variables socioculturales, producto de los diagnósticos institucionales, que les permita conocer y caracterizar a los estudiantes de los distintos programas de estudio en el marco de los nuevos requerimientos de la ley de carrera docente chilena de tal manera que contribuya a promover la equidad y calidad de la educación universitaria al considerar la diversidad y heterogeneidad en las carreras de pedagogía dado que la capacidad de razonar científicamente se posiciona como un factor relevante en el rendimiento de los estudiantes y el desarrollo de los ciudadanos del siglo XXI.

\section{AGRADECIMIENTOS}

Este estudio ha sido realizado y financiado con el patrocinio de los Proyectos de Mejoramiento Institucional PMI USA1503 y PMI USA1502: "Plan de armonización curricular para fortalecer la implementación, seguimiento y evaluación de las innovaciones en los planes de estudio de carreras y programas de la Universidad de Santiago de Chile". Los autores agradecen el aporte realizado por la Dra. Carla Hernández durante el desarrollo del artículo. SR y ST de la "Universidad de Santiago de Chile, USACH. Agradecimientos DICYT 031941RR y 031931TF, Vicerrectoría de investigación, Desarrollo e innovación". 


\section{REFERENCIAS}

Al-Zoubi, T., El-Shara, I., y Al-Salam, M. K., The Scientific Reasoning Level of Students' in the Faculty of Science in Alhussein Bin Talal University and its Affection of Gender, Teaching Level, and Specialization, An-Najah University Journal for Research - Humanities, 23(2), 401-437(2009).

Ausubel, D.P, Novak, J. D., y Hanesian, H., Educational Psychology: A Cognitive View, 2a Ed., Holt, Rinehart, \& Winston, New York, USA (1978).

Ates, S. y Cataloglu, E., The Effects of Students' Reasoning Abilities on Conceptual Understandings and Problem-Solving Skills in Introductory Mechanics. https://doi.org/10.1088/0143-0807/28/6/013, European Journal of Physics, 28(6), 11611171 (2007).

Ávalos, B., La Formación Inicial Docente en Chile: Tensiones entre Políticas de Apoyo y Control. https://doi.org/10.4067/S0718-07052014000200002, Estudios Pedagógicos, 40 (Especial), 11-28 (2014).

Bao, L., Xiao, Y., Koenig, K., y Han, J., Validity Evaluation of the Lawson Classroom Test of Scientific Reasoning. https://doi.org/10.1103/PhysRevPhysEducRes.14.020106, Physical Review Physics Education Research, 14(2), 0201061 (2018).

Cerda, G.A, Salcedo, P.A, y otros 2 autores, Futuros Profesores de Matemáticas: Rol de la Disponibilidad Léxica, Esquemas de Razonamiento Formal en Logros Académicos Durante su Formación Inicial. https://dx.doi.org/10.4067/S0718-50062017000100005, Formación Universitaria, 10(1), 33-46. (2017).

Coletta, V. P. y Phillips, J. A. Interpreting FCl Scores: Normalized Gain, Pre Instruction Scores, and Scientific Reasoning Ability, American Journal of Physics, 73(12), 1172-1182 (2005).

Coletta, V. P., Phillips, J. A., y Steinert, J. J., Why You Should Measure Your Students' Reasoning Ability. https://doi.org/10.1119/1.2715422, The Physics Teacher, 45(4), 235-238 (2007).

Coletta, V. P., Phillips, J. A., y otros 4 autores, FCI Normalized Gain, Scientific Reasoning Ability, Thinking in Physics, and Gender Affects. https://doi.org/10.1063/1.3679984, 1413, 23-26 (2012).

Decreto 239. Diario Oficial de la República de Chile, Santiago, Chile 17 de Noviembre, (2016).

Ding, L., Wei, X., y Liu, X., Variations in University Students' Scientific Reasoning Skills across Majors, Years, and Types of Institutions. https://doi.org/10.1007/s11165-015-9473-y, Research in Science Education, 46(5), 613-632 (2016).

Fisher, F., Chinn, C., y otros 2 autores. Scientific Reasoning and Argumentation: The Roles of Domain-Specific and Domain-General Knowledge, Routledge, New York, USA (2018).

Gunhaart, A., y Srisawasdi, N., Effect of Integrated Computer-Based Laboratory Environment on Students' Physics Conceptual Learning of Sound Wave Properties. https://doi.org/10.1016/j.sbspro.2012.06.510 Procedia-Social and Behavioral Sciences, 46, 5750-5755 (2012).

Hernández, R., Fernández, C., y Baptista, P., Metodología de la Investigación, 6a Ed., McGraw-Hill, D.F., México (2014). Ibrahim, B., Ding, L., Mollohan, K. y Stammen, A., Scientific Reasoning: Theory Evidence Coordination in Physics-based and Non-physics-based Tasks. https://doi.org/10.1080/10288457.2015.1108570, African Journal of Research in Mathematics, Science and Technology Education, 20(2), 93-105 (2016).

Infante, M., Desafíos a la Formación Docente: Inclusión Educativa. https://doi.org/10.4067/s0718-07052010000100016, Estudios Pedagógicos, 36, 287-297 (2009).

Labarrere, A., Zona de Desarrollo Próximo como Eje del Desarrollo de los Estudiantes: de la Ayuda a la Colaboración. https://doi.org/10.18774/448x.2016.13.293, Summa Psicológica UST, 13(1), 45-56 (2016).

Lawson, A. E., Teaching Inquiry Science in Middle and Secondary Schools, 1a Ed., Sage. Los Angeles, USA (2010).

Lawson, A. E., Banks, D.L. y Logvin, M., Self-efficacy, Reasoning Ability and Achievement in College Biology. https://doi.org/10.1002/tea.20172, Journal of Research in Science Teaching, 44, 706-724 (2007).

Ley № 20.129. Diario Oficial de la República de Chile, Santiago, Chile, 29 de mayo, (2018).

Liao, Y.W., y She, H.C., Enhancing Eighth Grade Students' Scientific Conceptual Change and Scientific Reasoning through a Web-based Learning Program. Educational Technology \& Society, 12(4), 228-240 (2009).

Lobato, C., y Madinabeitia, A., Perfiles Motivacionales del Profesorado ante la Formación en Metodologías Activas en la Universidad, https://dx.doi.org/10.4067/S0718-50062011000100006, Formación universitaria, 4(1), 37-48. (2011).

Moore, J. C., Teaching Science Thinking: Using Scientific Reasoning in The Classroom. Routledge,New York, USA (2019).

Osborne, J., Rafenelli, S., Kind, P. Toward a more coherent model for science education than the crosscutting concepts of the next generation science standards: the affordances of styles of reasoning. Journal of Research in Science Teaching, 55(7), 962-981 (2018).

Pérez de Landazábal, M.C., Fernández, y otros dos autores, Razonamiento y Destrezas Científicas de los Alumnos que Acceden a la Universidad. En Aprendizaje Activo de la Física Básica Universitaria. Benegas, J. Pérez de Landazábal M.C. y Otero, J. (eds.), 21-41. Andavira, España. (2013). 
Piaget, J., Science of Education and the Psychology of the Child. 1a Ed., Orion Press, New York, USA (1970).

Piraksaa, C., Srisawasdib, N., Koulc, R. Effect of Gender on Students' Scientific Reasoning Ability: A Case Study in

Thailand. Procedia-Social and Behavioral Sciences, 116(2014), 486-491. (2014).

Rodríguez, M., Mena, D., y Rubio, C., Razonamiento Científico y Conocimientos Conceptuales de Mecánica: Un Diagnóstico de Alumnos de Primer Ingreso a Licenciaturas en Ingeniería. https://doi.org/10.4067/S071850062010000500006, Formación universitaria, 3(5), 37-46 (2010).

Sjöström, J., y Eilks I., Reconsidering Different Visions of Scientific Literacy and Science Education Based on the Concept of Bildung; in Cognition, Metacognition, and Culture in STEM Education, by Dori Y., Mevarech Z., \& Baker D., 24, pp 6588, Springer, Dordrecht, Netherlands (2018).

Stammen, A. N., Malone, K. L., y Irving, K. E., Effects of Modeling Instruction Professional Development on Biology Teachers' Scientific Reasoning Skills. https://doi.org/10.3390/educsci8030119, Education Sciences, 8(3), 119 (2018).

UNESCO, Guía para Asegurar la Inclusión y la Equidad en la Educación. 1a Ed., Disponible en: https://unesdoc.unesco.org/ark:/48223/pf0000259592, UNESCO, Paris, Francia (2017).

Villarroel, V., y Bruna, D., Reflexiones en torno a las Competencias Genéricas en Educación Superior: Un Desafío Pendiente. Psicoperspectivas, 13(1), 23-34 (2014).

Weld, J., Stier, M., y Birren, J. M., The Development of a Novel Measure of Scientific Reasoning Growth among College Freshmen: The Constructive Inquiry Science Reasoning Skills Test. Research and Teaching, 40(4), 101-107 (2011).

Zárate-Rueda, R., Díaz-Orozco, S. P., y Ortiz-Guzmán, L., Educación Superior Inclusiva: Un Reto para las Prácticas Pedagógicas. https://doi.org/10.15359/ree.21-3.15, Revista Electrónica Educare, 21(3), 1-24 (2017).

Zimmerman, C., The Development of Scientific Thinking Skills in Elementary and Middle School. https://doi.org/10.1016/j.dr.2006.12.001, Developmental Review, 27, 172-223 (2007). 